\title{
ParMap, an algorithm for the identification of small genomic insertions and deletions in nextgen sequencing data
}

\author{
Hossein Khiabanian*1,2, Pieter Van Vlierberghe33, Teresa Palomero3,4, Adolfo A Ferrando3,4,5 and Raul Rabadan1,2
}

\begin{abstract}
Background: Next-generation sequencing produces high-throughput data, albeit with greater error and shorter reads than traditional Sanger sequencing methods. This complicates the detection of genomic variations, especially, small insertions and deletions.

Findings: Here we describe ParMap, a statistical algorithm for the identification of complex genetic variants, such as small insertion and deletions, using partially mapped reads in nextgen sequencing data.

Conclusions: We report ParMap's successful application to the mutation analysis of chromosome X exome-captured leukemia DNA samples.
\end{abstract}

\section{Background}

One of the major technological advances in biology in the last few years has been the development of high throughput nextgen sequencing systems that produce gigabases of data in a single run, and allow an unbiased view of the whole genome without relying on prior knowledge about the disease-causing alterations. These ultradeep sequencing technologies produce large amounts of sequence data, which increase the sequencing depth and allow for better statistics in calling various genomic variations. However, they do so at the cost of reducing the read length and increasing the error rate relative to traditional Sanger sequencing. Thus, the development of efficient statistical and computational methods for the high confidence call of genomic variants is needed for the analysis of these high throughput datasets.

At this point, the detection of single mutations and large copy number variations using deep sequencing data is fairly straight forward $[1,2]$, whereas the identification of small (less than 10 nucleotides) insertions and deletions is more challenging. A few algorithms have been developed for detecting complex genomic variants, such as structural variations and insertions and deletions,

* Correspondence: hossein@c2b2.columbia.edu

1 Department of Biomedical Informatics, Columbia University College of Physicians and Surgeons, New York, NY, USA

Full list of author information is available at the end of the article using mate-pair or paired-end reads [3,4], however, identifying small insertion/deletions in fragment (single-end) data has proved to be very difficult. Mapping algorithms that are designed for very short reads have to assign large penalties for introducing gaps in the middle of the alignment in order to map the majority of the reads efficiently. Conversely, decreasing the gap penalties, increases the number of reads that are mapped with low confidence. However, these methods can partially map reads to a reference genome with gaps at the either end, without significantly reducing the alignment score. Although these gaps may be caused by systematic errors in the sequencing and mapping processes, we hypothesized that gaps that appear in multiple reads at a given position on the genome may reflect the presence of a more complex genomic variant such as insertion, deletion, or multiple base changes. However, this algorithm is not capable of detecting structural variants.

Following this principle, we aimed to develop a procedure for identifying small genomic insertions and deletions with high confidence and built an algorithm (ParMap) capable of producing a list of candidates (along with their nucleotide sequence), through statistical analysis of partially mapped reads (Figure 1). Specifically, ParMap calculates a measure based on the number of reads that only cover the positions adjacent to a gap without covering their neighboring positions in the direction of

(c) 2010 Khiabanian et al; licensee BioMed Central Ltd. This is an open access article distributed under the terms of the Creative Com:-11ed Central mons Attribution License (http://creativecommons.org/licenses/by/2.0), which permits unrestricted use, distribution, and reproduction in any medium, provided the original work is properly cited. 
(1) GAATGTGGACAGTTACTAATATCTGAAAACCAGAAGGTGGCAGCGCACCA (2) GAATGTGGACAGTTACTAATATCTGAAAACCAGAAGGTGGCAGCGAGGCA (3) GAATGTGGACAGTTACTAATATCTGAAAACCAGAAGGTGGCAGCG

$$
\overrightarrow{5^{\prime} \quad 3^{\prime}}
$$$$
\text { p }
$$

Figure 1 ParMap employs reads that are partially mapped to a reference genome to identify genomic variations. These variations include small insertions and deletions of less than 10 nucleotides. When the sequenced read (line 2 ) is mapped to the reference genome (line 1), the unmatched bases are marked as gaps (line 3), adjacent to position $p$ (Methods).

the gap, to identify the possible locations of genomic insertions or deletions (Figure 2 and Methods).

\section{Results and discussion}

To test the ability of this method to detect novel complex genomic variants, such as insertion, deletion, and multiple base changes, we applied ParMap to the analysis of SOLiD 3 chromosome $\mathrm{X}$ exome sequencing data from 12 T-cell acute lymphoblastic leukemia (T-ALL) DNA samples [5]. In this experiment leukemia DNA samples were fragmented and ligated to adapters to generate SOLiD sequencing libraries, which were amplified and subsequently enriched in chromosome $\mathrm{X}$ exonic sequences using the SureSelect Target Enrichment System [6], a platform which targets 5,217 exonic regions encompassing 3,045,708 nucleotides in the $\mathrm{X}$ chromosome. The Chromosome X exome captured DNA samples were sequenced with the Applied Biosystems SOLiD 3 platform using $1 / 8^{\text {th }}$ of sequencing slide per sample to produced a total of 105,302,787 fifty-base long fragment reads. The SOLiD platform employs a ligation based



Figure 2 A measure based on the number of reads that only cover position $p$ without covering its neighboring position $(p \pm 1)$ in the direction of the gap is calculated. In other words, we find the ratio of the intersection (orange area) and the union (yellow and red areas) of the two sets of reads that cover $p$ or $p \pm 1$ (Figure 1). Here, $N(p), N(p \pm$ $1)$, and $N(p \& p \pm 1)$ are the number of reads that cover position $p$, position $p \pm 1$, and both positions, respectively (Methods). chemistry and a two-base encoding system, where each pair of nucleotides is reported with a different color, depending on the first base within the pair. Therefore, to call a single-base change relative to the reference sequence in nucleotide-space, two consecutive colorspace mismatches must be observed. Single color-space mismatches solely report errors in the reads [7].

To ensure an optimum mapping of these sequencing results, we created a reference sequence containing all chromosome $\mathrm{X}$ capture targeted regions plus adjacent 50 flanking bases using the March 2006 human reference sequence assembly (hg18). We used the SHRiMP algorithm with its default parameters for mapping the reads [8]. For further analysis of our dataset, we only included the reads with a maximum number of two color-space mismatches that were uniquely mapped to the reference genome (approximately $31 \%$ of the raw reads). An average $90.1 \%$ of the reference genome was found covered in the samples, with a mean depth of 42 per base. Less restrictive filtering increased the false positive rate of candidate genomic variants without a significant increase in the coverage.

We created a candidate list of single-base variants for which a minimum of 3 reads (consistent with $1 \%$ estimated error rate of this particular run) should map to the candidate's position, with more than $75 \%$ of them calling the nucleotide change. T-ALL samples such as the ones analyzed in this series contain over $80 \%$ tumor cells, however a small fraction of contaminating normal cells is expected. Because of the possibility of this contamination, we did not enforce a $100 \%$ consensus among the reads. To identify genetic alterations with the most direct impact in gene function we focused on the analysis of non-synonymous changes in the captured exons.

In this analysis we noticed numerous systematic errors that cannot be corrected by increasing the sequencing depth, i.e. genomic variants that are reported systematically beyond the statistical expectations from the estimated error rates. These systematic errors arise from presequencing operations, ligation-based sequencing, mapping, and specific genomic variants in the reference genome. Due to these redundancies in the capture or mapping, some variations appear in most of the samples. For instance, an identical single-base variant in the exonic region of the SLC6A8 gene was found in 10 samples, within $73 \%$ of the reads, beyond the statistical expectations from the estimated error rates. Therefore, we removed the positions that seemed prone to systematic errors by combining the candidate lists from all the samples and only keeping the genomic variations that occur in less than 3 samples at a given position. None of the variations that were found in more than 3 samples were validated via Sanger sequencing. 
Within the 12 samples analyzed, we identified 66 exonic non-synonymous single-base variant candidates, which were not listed as already known polymorphisms in the human genome [9]. Overall, 61/66 (92\%) of these candidates were confirmed via Sanger sequencing (Table 1). Applying the same filtering criteria results in a similar number of candidates when another alignment algorithm, Corona-Lite [10], is run on our dataset. This indicates that the number of significant candidates does not depend on the mapping method.

Next, we applied the ParMap algorithm to our dataset to identify possible more complex variants such as small insertions and deletions. Following on the selection of candidate variants using ParMap filtering criteria, we selected the ones that were detected in a single sample each. In this analysis, we found a high prevalence of systematic errors in intron-exon boundaries, which may reflect impaired ligation-based sequencing in these positions. Therefore, candidate variants located in intronexon boundaries were discarded and excluded from further analysis. ParMap identified a total of 7 candidate complex variants (Table 1). Using Sanger sequencing of PCR products encompassing these sequences, we confirmed four indels in four different samples, including two genomic deletions of 3 and 6 nucleotides and two genomic insertions of 5 and 3 nucleotides. Notably, the genomic sequences identified in each of these two insertions matched the predicted sequence variant in the ParMap's results.

\section{Conclusions}

We have demonstrated the successful identification of high confidence genomic variants in nextgen sequencing data using a combination of single nucleotide analysis and ParMap. (Currently, ParMap is available for download upon request from the authors.) Overall, 89\% of our candidate variants were experimentally validated in this series. ParMap may enhance the identification of elusive complex genetic variants such as small insertions and deletions in nextgen sequencing data, taking advantage of partially mapped reads that might otherwise be discarded.

\section{Methods}

In addition to the completely mapped reads and reads reporting single-base changes, the dataset includes partially mapped reads, with the unmatched positions marked as gaps. These reads either start or end with a gap region that is as long as $20 \%$ of the length of the read (Figure 1). ParMap makes use of such reads and for any position $p$ that is adjacent to a gap region and is not the starting or ending position of an exon, calculates the following quantities:

1. $N(p)$ : The number of reads that cover position $p$.

2. $N(p \pm 1)$ : The number of reads that cover position $p$ \pm 1 . (Plus, if $p$ is the position after the gap and minus, if $p$ is the position before the gap, in the direction of the positive strand.)

3. $N(p \& p \pm 1)$ : The number of reads that cover both positions of $p$ and $p \pm 1$.

We define

$$
r=\frac{N(p \& p \pm 1)}{N(p)+N(p \pm 1)-N(p \& p \pm 1)}
$$

which is an inverse measure of the number of reads that only cover the position $p$ without covering its neighboring position in the direction of the gap (Figure 2). Therefore, the smaller the value of $r$, the higher the chance for the gap to be due to a real change in the sequenced genome. Moreover, because the reads in which position $p$ is adjacent to a gap region are already collected, referring back to each read prior to the mapping, the genomic sequence of the gap region can be extracted.

We apply the following criteria to produce a list of candidates: the value of $r$ should be less that 0.35 and at least 5 reads should map to $p$ adjacent to a gap, reporting a consensus sequence for it. To reduce the systematic errors due to mapping artifacts, we remove the candidates whose gap regions cover the already known polymorphisms of the human genome. We experimentally observed that less restrictive criteria increased the number of false positives.

Table 1: Summary of the efficiency of our identification methods.

\begin{tabular}{|c|c|c|c|}
\hline & Number of Genomic Variation Candidates & Number of Confirmed Candidates & Percentage \\
\hline Singe-base Analysis & 66 & 61 & $92 \%$ \\
\hline ParMap & 7 & 4 & $57 \%$ \\
\hline Total & 73 & 65 & $89 \%$ \\
\hline
\end{tabular}

In total, $89 \%$ of our candidates were confirmed via Sanger sequencing. 


\section{Competing interests}

The authors declare that they have no competing interests.

\section{Authors' contributions}

HK and RR conceived the ParMap algorithm. HK coded the algorithm and applied it to high throughput sequencing data obtained by TP at the Genomics Technologies Shared Resource of Columbia University. Validation was performed by PVV in AF laboratory. All authors contributed to the development of the method and wrote the paper.

\section{Acknowledgements}

This work was supported by awards from the Northeast Biodefence Center (U54-Al057158 to RR), the National Library of Medicine (1R01LM010140-01 to RR), the National Institutes of Health (R01CA120196 and R01CA129382 to AF); the Rally Across America Foundation (AF); the Swim Across America Foundation (AF) and the Golfers Against Cancer Foundation (AF). PW is supported by a postdoc grant from the Fund for Scientific Research (FWO) Flanders. TP is a recipient of a Young Investigator Award from the Alex's Lemonade Stand Foundation, and AF is a Leukemia \& Lymphoma Society Scholar. We are especially grateful to Vladimir Trifonov for many insightful discussions.

\section{Author Details}

'Department of Biomedical Informatics, Columbia University College of Physicians and Surgeons, New York, NY, USA, ${ }^{2}$ Center for Computational Biology and Bioinformatics, Columbia University College of Physicians and Surgeons, New York, NY, USA, 3Institute for Cancer Genetics, Columbia University College of Physicians and Surgeons, New York, NY, USA, 4Department of Pathology, Columbia University College of Physicians and Surgeons, New York, NY, USA and 5 Department of Pediatrics, Columbia University College of Physicians and Surgeons, New York, NY, USA

Received: 29 January 2010 Accepted: 27 May 2010

Published: 27 May 2010

\section{References}

1. Shendure J, Ji H: Next-generation DNA sequencing. Nat. Biotechnol 2008, 26:1135-45.

2. McPherson JD: Next-generation gap. Nat. Methods 2009, 6:S2-S5.

3. Medvedev P, Stanciu M, Brudno M: Computational methods for discovering structural variation with next-generation sequencing. Nat Methods 2009, 6:S13-S20.

4. Dalca AV, Brudno M: Genome variation discovery with high-throughput sequencing data. Brief Bioinform 2010, 1:3-14.

5. Van Vlierberghe P, Palomero T, Khiabanian H, Van der Meulen J, Castillo M, Van Roy N, De Moerloose B, Philippé J, González-García S, Toribio ML, Taghon T, Zuurbier L, Cauwelier B, Harrison CJ, Schwab C, Pisecker M, Strehl S, Langerak AW, Gecz J, Sonneveld E, Pieters R, Paietta E, Rowe JM, Wiernik PH, Benoit Y, Soulier J, Poppe B, Yao X, Cordon-Cardo C, Meijerink J, Rabadan R, Speleman F, Ferrando A: PHF6 mutations in T-cell acute lymphoblastic leukemia. Nat Genetics 2010, 42:338-42.

6. Gnirke A, Melnikov A, Maguire J, Rogov P, LeProust EM, Brockman W, Fennell T, Giannoukos G, Fisher S, Russ C, Gabriel S, Jaffe DB, Lander ES, Nusbaum C: Solution hybrid selection with ultra-long oligonucleotides for massively parallel targeted sequencing. Nat Biotechnol 2009, 27:182-9.

7. Homer N, Merriman B, Nelson SF: Local alignment of two-base encoded DNA sequence. BMC Bioinformatics 2009, 10:175.

8. Rumble SM, Lacroute P, Dalca AV, Fiume M, Sidow A, Brudno M: SHRiMP: accurate mapping of short color-space reads. PLoS Comput Biol 2009, 5:e1000386.

9. Kuhn RM, Karolchik D, Zweig AS, Wang T, Smith KE, Rosenbloom KR, Rhead B, Raney BJ, Pohl A, Pheasant M, Meyer L, Hsu F, Hinrichs AS, Harte RA, Giardine B, Fujita P, Diekhans M, Dreszer T, Clawson H, Barber GP, Haussler D, Kent WJ: The UCSC Genome Browser Database: update 2009. Nucleic Acids Res 2009, 37:D755-D761.

10. McKernan KJ, Peckham HE, Costa GL, McLaughlin SF, Fu Y, Tsung EF, Clouser CR, Duncan C, Ichikawa JK, Lee CC, Zhang Z, Ranade SS, Dimalanta ET, Hyland FC, Sokolsky TD, Zhang L, Sheridan A, Fu H, Hendrickson CL, Li B, Kotler L, Stuart JR, Malek JA, Manning JM, Antipova AA, Perez DS, Moore MP, Hayashibara KC, Lyons MR, Beaudoin RE, Coleman BE, Laptewicz MW, Sannicandro AE, Rhodes MD, Gottimukkala RK, Yang S, Bafna V, Bashir A,
MacBride A, Alkan C, Kidd JM, Eichler EE, Reese MG, De La Vega FM, Blanchard AP: Sequence and structural variation in a human genome uncovered by short-read, massively parallel ligation sequencing using two-base encoding. Genome Res 2009, 19:1527-41.

doi: $10.1186 / 1756-0500-3-147$

Cite this article as: Khiabanian et al., ParMap, an algorithm for the identification of small genomic insertions and deletions in nextgen sequencing data BMC Research Notes 2010, 3:147

\section{Submit your next manuscript to BioMed Central and take full advantage of:}

- Convenient online submission

- Thorough peer review

- No space constraints or color figure charges

- Immediate publication on acceptance

- Inclusion in PubMed, CAS, Scopus and Google Scholar

- Research which is freely available for redistribution

Submit your manuscript at www.biomedcentral.com/submit
C) Biomed Central 